\title{
Band Structure Calculations of Photonic Crystals with Dispersive Materials
}

\author{
Jyh-Yang Wang ${ }^{1}$, Yean-Woei Kiang ${ }^{1,2,3}$ and C. C. Yang ${ }^{2,3,4}$ \\ ${ }^{1}$ Graduate Institute of Communication Engineering, National Taiwan University, \\ 1, Roosevelt Road, Sec. 4, Taipei, Taiwan \\ (phone) 886-2-23635251ext.438 (fax) 886-2-23652637 (E-mail) ywkiang@ew.ee.ntu.edu.tw \\ ${ }^{2}$ Department of Electrical Engineering, National Taiwan University, Taipei, Taiwan \\ ${ }^{3}$ Graduate Institute of Electro-Optical Engineering, National Taiwan University, Taipei, Taiwan \\ ${ }^{4}$ Graduate Institute of Electronics Engineering, National Taiwan University, Taipei, Taiwan
}

\begin{abstract}
The band structures of dispersive photonic crystals are numerically investigated. Based on the finite-difference time-domain (FDTD) method with some auxiliary variables, time-stepping formulas are derived to compute the evolution of electromagnetic fields. The photonic crystals made of dispersive materials can be described either by the Lorentzian model or by the Drude model. In this paper, we describe how to use the time domain technique to calculate the dispersion relation of photonic crystals and then introduce the recursive convolution scheme to treat the photonic crystals with frequency-dependent materials. Band structures of photonic crystals without dispersion are calculated with the conventional FDTD time-stepping formulas and those with Lorentzian type dispersion and Drude type dispersion are also calculated with the recursive convolution scheme.
\end{abstract}

Keywords: photonic crystals, dispersive materials, FDTD.

\section{INTRODUCTION}

In the past few years, the photonic crystal, which is of structure with periodicity, has attracted wide attention in both physics and engineering communities. It has a possibility of offering some frequency bands, in which electromagnetic wave can not propagate in all directions, i.e., photonic band gaps. The photonic band gap is the optical analogy of the band gap of electronic system with periodic lattice potential [1], [2]. The behavior of the band structure is the most important thing we are concerned about in this kind of problems. For nondispersive photonic crystals, the plane wave expansion method [3] and the FDTD [4], [5] method are widely used to obtain the band structures. People use the FDTD because it is suitable to treat the problems of complex geometries. On the other hand, the plane wave expansion method has also been used because it is a more direct way for calculating frequency domain quantities. But for dispersive photonic crystal, the eigenvalue problems derived from the plane wave expansion are not in a standard form and are difficult to solve. Fortunately, for some dispersion types, the convolution calculation can be simplified into a recursive form, making the calculation possible. The Lorentzian model for semiconductors and the Drude model for cold plasma are the most often used models. Both of them have recursive forms for the convolution between the displacement vector $\vec{D}$ and electric field $\vec{E}$. We will introduce the recursive convolution scheme in the next section.

\section{BAND STRUCTURE CALCULATION USING FDTD}

As regards the band structure calculations, we choose the FDTD method for our simulations. Note that the FDTD method is more convenient than the plane wave expansion method in modeling complicated structures or those with defects. Most importantly, the FDTD is a method of order $\mathrm{N}$ while the plane wave expansion is the one of order $\mathrm{N}^{3}$. It can save more computation time when calculating with FDTD.

In an FDTD algorithm, the computation domain is discretized in time and space and the Maxwell's equations are described in finite difference instead of derivative. Provided the initial conditions and boundary conditions are specified, the fields at all space points in any time after the initial time can be obtained. It is necessary to properly set the boundary conditions and the initial fields. Since the structure of a photonic crystal is periodic, a unit cell of lattice is naturally chosen as the finite computation domain. It is proved that in an infinite periodic structure the electromagnetic field must satisfy the 
Bloch condition, i.e., the field can be written in the following Bloch form [1]:

$$
\vec{E}(\vec{r})=e^{-j \vec{k} \cdot \vec{r}} \vec{e}(\vec{r}), \vec{H}(\vec{r})=e^{-j \vec{k} \cdot \vec{r}} \vec{h}(\vec{r}),
$$

where $\vec{e}(\vec{r})$ and $\vec{h}(\vec{r})$ are periodic functions in space and $\vec{k}$ is the prescribed wavevector. Consequently, the boundary conditions are as follows:

where $\vec{T}$ is the lattice vector.

$$
\vec{E}(\vec{r}+\vec{T})=e^{-j \vec{k} \cdot \vec{T}} \vec{E}(\vec{r}), \vec{H}(\vec{r}+\vec{T})=e^{-j \bar{k} \cdot \vec{T}} \vec{H}(\vec{r}),
$$

For most dispersive materials, the frequency dependence of the complex relative permittivity can be described by the following form:

$$
\varepsilon_{\omega r}(\omega)=\varepsilon_{\infty}+\chi_{\omega}(\omega)
$$

where $\varepsilon_{\infty} \varepsilon_{0}$ is the permittivity at infinite frequency. The relationship between $\vec{D}$ and $\vec{E}$ in the time domain can be written in a convolution integral

$$
\vec{D}(t)=\varepsilon_{\infty} \varepsilon_{0} \vec{E}(t)+\varepsilon_{0} \int_{0}^{t} \vec{E}(t-\tau) \chi_{t}(\tau) d \tau,
$$

where $\chi_{t}(\tau)$, the time domain susceptibility, is the Fourier transform of $\chi_{\omega}(\omega)$. By discretizing the continuous time expression of (4) and assuming that all field quantities are constants over each time interval, $\Delta t,(4)$ can be simplified to

$$
\vec{D}(t) \approx \vec{D}(n \Delta t)=\vec{D}^{n}=\varepsilon_{\infty} \varepsilon_{0} \vec{E}^{n}+\varepsilon_{0} \sum_{m=0}^{n-1} \vec{E}^{n-m} \int_{m \Delta t}^{(m+1) \Delta t} \chi_{t}(\tau) d \tau
$$

Substituting (5) into the Maxwell's equations, we obtain the time-stepping formula [6] for $\vec{E}$ :

$$
\vec{E}^{n+1}=\frac{\varepsilon_{\infty}-\frac{\sigma \Delta t}{2 \varepsilon_{0}}}{\frac{\sigma \Delta t}{2 \varepsilon_{0}}+\varepsilon_{\infty}+\chi^{0}} \vec{E}^{n}+\frac{1}{\frac{\sigma \Delta t}{2 \varepsilon_{0}}+\varepsilon_{\infty}+\chi^{0}} \vec{\Psi}^{n}+\frac{\frac{\Delta t}{\varepsilon_{0}}}{\frac{\sigma \Delta t}{2 \varepsilon_{0}}+\varepsilon_{\infty}+\chi^{0}} \nabla \times \vec{H}^{n+\frac{1}{2}}-\frac{\frac{\Delta t}{\varepsilon_{0}}}{\frac{\sigma \Delta t}{2 \varepsilon_{0}}+\varepsilon_{\infty}+\chi^{0}} \vec{J}^{n+\frac{1}{2}}
$$

where $\vec{\Psi}^{n}=\sum_{m=0}^{n-1} \vec{E}^{n-m}\left(\chi^{m}-\chi^{m+1}\right)$ and $\chi^{m}=\int_{m \Delta t}^{(m+1) \Delta t} \chi_{t}(\tau) d \tau$.

For Drude type dispersion, $\varepsilon_{\infty}=1$ and the susceptibility in frequency domain is $\chi_{\omega}(\omega)=\frac{\omega_{p}{ }^{2}}{\omega\left(j v_{c}-\omega\right)}$, corresponding to the time domain susceptibility $\chi_{t}(t)=\frac{\omega_{p}{ }^{2}}{v_{c}}\left(1-e^{-v_{c} t}\right) U(t)$, where $\omega_{p}$ is the angular plasma frequency and $\nu_{c}$ is the collision frequency. In such a case, $\bar{\Psi}^{n}$ can be simplified to a recursive form:

$$
\vec{\Psi}^{n}=-\vec{E}^{n} \frac{\omega_{p}{ }^{2}}{v_{c}{ }^{2}}\left(1-e^{-v_{c} \Delta t}\right)^{2}+e^{-v_{c} \Delta t} \bar{\Psi}^{n-1}
$$

For Lorentzian type dispersion, $\chi_{\omega}(\omega)$ is given by $\chi_{\omega}(\omega)=\left(\varepsilon_{s}-\varepsilon_{\infty}\right) \frac{\omega_{0}^{2}}{\omega_{0}^{2}+2 j \omega \delta-\omega^{2}}$, where $\omega_{0}$ is the resonant frequency, $\delta$ the damping frequency, and $\varepsilon_{s} \varepsilon_{0}$ the static permittivity. The recursive form of $\hat{\bar{\Psi}}^{n}$ can also be obtained in a similar way. Note that $\hat{\bar{\Psi}}^{n}$ is defined so that $\vec{\Psi}^{n}=\operatorname{Re}\left(\hat{\bar{\Psi}}^{n}\right)$.

As to the initial setting of the fields, we can simply choose a Gaussian pulse, which contains the frequencies we are interested in, to excite the eigenmodes. At each space point, we execute the field evolution until the initial transient dies out and the quasi-steady state is attained. Then time sequence field at a specified space point is selected. We use the fast Fourier transform (FFT) to obtain the spectrum of this time sequence. The peak locations of the spectrum represent the desired eigenfrequencies. However, the initial setting of the fields described above does not satisfy the Bloch condition resulting in the failure of applying the periodic boundary condition (2). It would take much time to reach the quasi-steady state in this situation. If we choose an initial field distribution satisfying the Bloch condition to replace a Gaussian pulse, the situation will be improved. Note that all the calculation procedures we discussed above are for one certain $\vec{k}$ used in the periodic boundary condition. Besides, it would be better to take the average of the spectra at several space points randomly chosen. 


\section{SIMULATION RESULTS}

The photonic crystals of interest are 2-D structures consisting of a square array of dielectric rods surrounded by air, as shown in Fig. 1. The lattice constant is $a$ and the radius of dielectric rods is $r$. The first Brillouin zone of this photonic crystal is shown in Fig. 2. The three special point, $\Gamma, \mathrm{X}$, and $\mathrm{M}$ correspond to $\vec{k}=0, \vec{k}=\hat{x} \pi / a$, and $\vec{k}=\hat{x} \pi / a+\hat{y} \pi / a$, respectively. Figure 3 shows the TM and TE simulated band structures of the nondispersive photonic crystal. The radius of dielectric rods is $r=0.2 a$ with the dielectric constant being 3 . Here, the rods are assumed to be lossless. Because of the weak index contrast, there exists no band gap in either TM or TE case. The simulated band structures of the photonic crystal with Lorentzian type dispersion are shown in Fig. 4. Here, the radius of the rods are the same as that in dispersion-free case and other parameters are $\omega_{0} a /(2 \pi c)=0.67, \delta=0.01 \omega_{0}, \varepsilon_{s}=3.1$ and $\varepsilon_{\infty}=2.9$. In this case, the variation in the relative permittivity of rods is weak and the band structures look like those in the dispersion-free case. However, some bands near the resonant frequency partially disappear due to the loss associated with dispersion. Figure 5 also shows the band structures of another Lorentzian material with stronger dispersion in which the same parameters are used except $\varepsilon_{s}=4$ and $\varepsilon_{\infty}=2$. Here, due to the increased variation in the relative permittivity, the band structures are obviously different from those shown in Fig. 3. Besides, the bands near the resonant frequency tend to disappear more resulting from the larger loss. The band structures for Drude type dispersion are shown in Fig. 6 with parameters $r=0.467 a, \omega_{p} a /(2 \pi c)=1$ and $\nu_{c}=0.01 \omega_{p}$. There exists no band in the low frequency range for the TM case where the electric field is parallel to the rods, consisting with the fact that the loss in the rods rapidly increases as the frequency approaches zero.

\section{CONCLUSIONS}

In summary, a recursive convolution FDTD method has been used to compute the band structures of photonic crystals with dispersive materials. The procedures of band structure calculation include using the FDTD to obtain time sequences at several space points, taking the average of their spectra, and picking out the peak locations as the desired eigenfrequencies. The TM and TE modes are calculated for both Lorentzian and Drude type materials. In either case, the results show that the frequency-dependent dielectric constant and the associated loss may affect the band structure considerably. However, it must be pointed out that the transmission calculation would be of more practical significance, which is under current study.

\section{ACKNOWLEDGEMENT}

This research was supported by National Science Council, The Republic of China, under the grants of NSC 90-2215-E-002-029, NSC 90-2212-M-002-052, NSC 90-2215-E-002-027, and NSC 90-2215-E-002-041.

\section{REFERENCES}

[1] J. D. Joannopoulos, R. D. Mead, and J. N. Winn, Photonic Crystals: Molding the Flow of Light, Princeton University Press, Princeton, 1995.

[2] K. Sakoda, Optical Properties of Photonic Crystals, Springer-Verlag, Berlin, 2001.

[3] M. Plihal, and A. A. Maradudin, "Photonic band structure of two-dimensional systems: the triangular lattice, Phys. Rev. B, Vol. 44, pp. 8565-8571, 1991.

[4] K. S. Yee, "Numerical solution of initial boundary value problems involving Maxwell's equations in isotropic media," IEEE Trans. Antennas Propagat., Vol. AP-14, pp. 302-307, 1966.

[5] M. Qiu and S. He, "A nonorthogonal finite-difference time-domain method for computing the band structure of a two-dimensional photonic crystal with dielectric and metallic inclusions," J. Appl. Phys., Vol. 87, pp. 8268-8275, 2000.

[6] K. S. Kunz and R. J. Luebbers, The Finite Difference Time Domain Method for Electromagnetics, CRC Press, Boca Raton, 1993. 


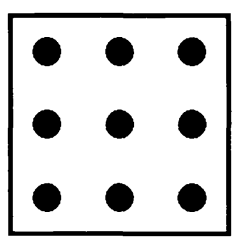

Fig. 1 A 2-D photonic crystal with a square lattice.

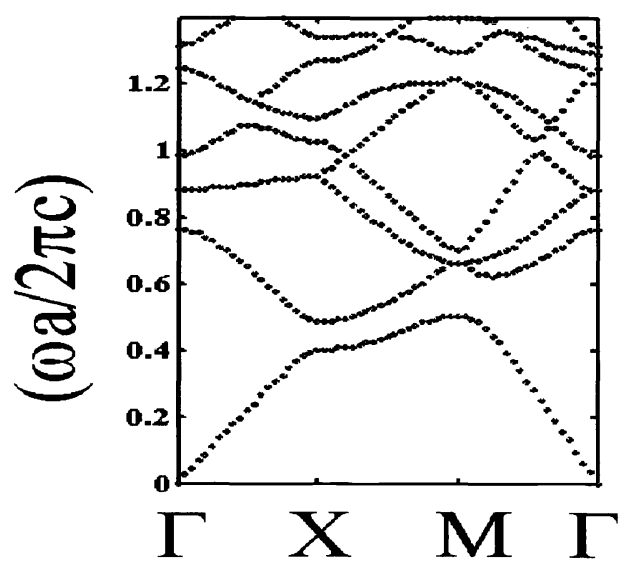

(a)

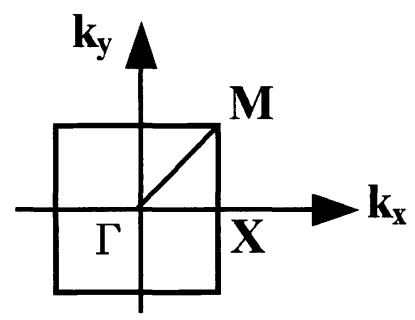

Fig. 2 The Brillouin zone of the square lattice.

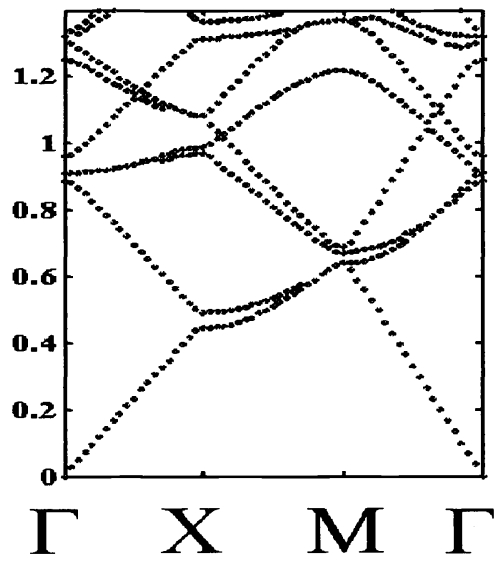

(b)

Fig. 3 The simulated band structures for a square array of dielectric rods with $r=0.2 a$. (a) TM modes; (b) TE modes. The dielectric constant of the nondispersive dielectric rods is $\varepsilon=3$.

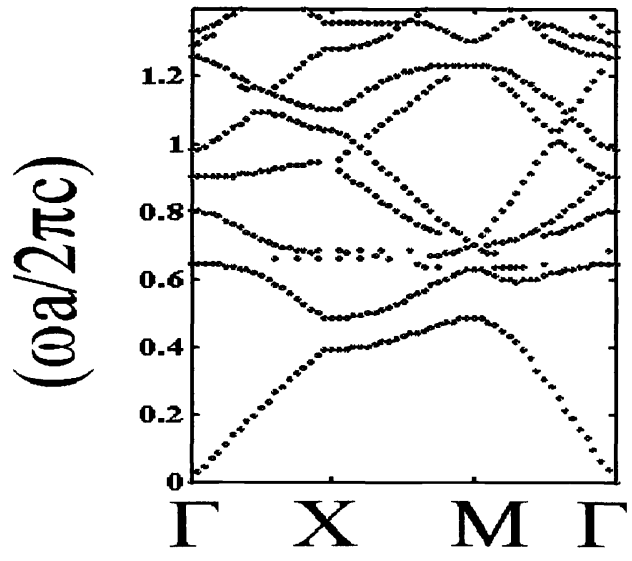

(a)

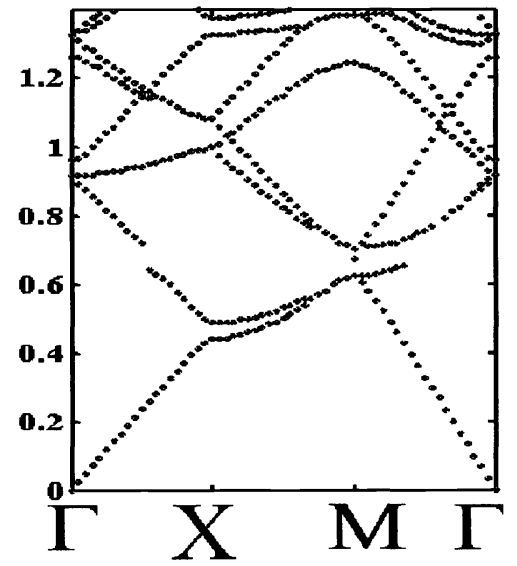

(b)

Fig. 4 The simulated band structures of the photonic crystal with Lorentzian type dispersion. (a) TM modes; (b) TE modes. The rods have a radius of $0.2 a$ with $\omega_{0} a /(2 \pi c)=0.67, \delta=0.01 \omega_{0}, \varepsilon_{s}=3.1$ and $\varepsilon_{\infty}=2.9$. 


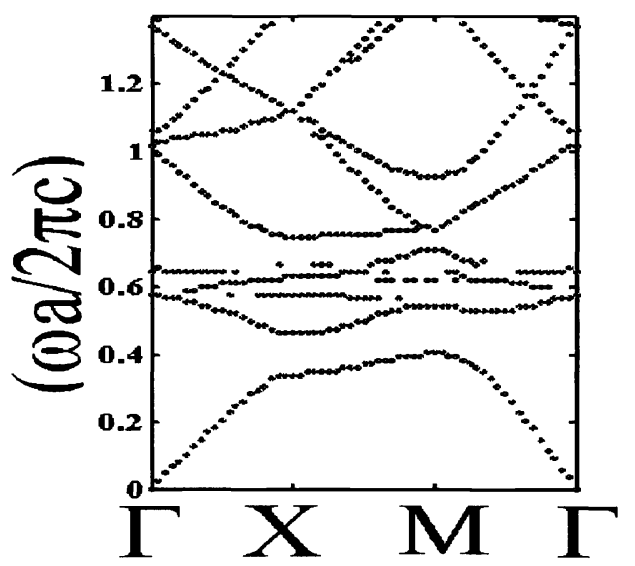

(a)

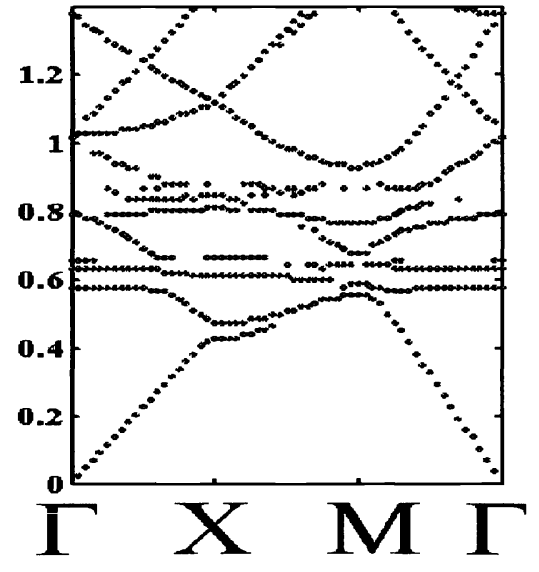

(b)

Fig. 5 The simulated band structures of the photonic crystal with Lorentzian type dispersion. (a) TM modes; (b) TE modes. The rods have a radius of $0.2 a$ with $\omega_{0} a /(2 \pi c)=0.67, \delta=0.01 \omega_{0}, \varepsilon_{s}=4$ and $\varepsilon_{\infty}=2$.

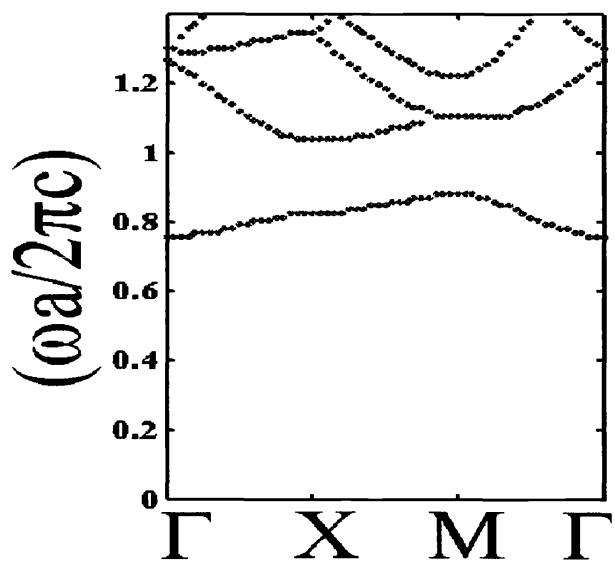

(a)

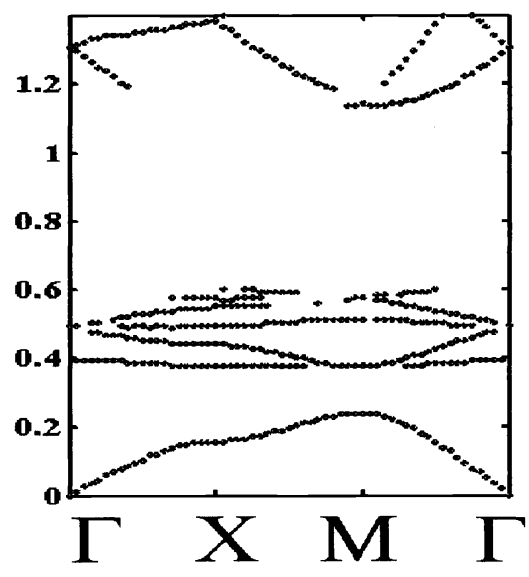

(b)

Fig. 6 The simulated band structures of the photonic crystal with Drude type dispersion. (a) TM modes; (b) TE modes. The rods have a radius of $0.467 a$ with $\omega_{p} a /(2 \pi c)=1$ and $\nu_{c}=0.01 \omega_{p}$ 